\title{
Hybrid Discrete Raman/EDFA Design for Broadband Optical Amplification in Metro WDM Systems
}

\author{
Lukasz Krzczanowicz, Ian Phillips, Md. Asif Iqbal, Mingming Tan, Paul Harper, and Wladek Forysiak \\ Aston Institute of Photonic Technologies, Aston University, B4 7ET Birmingham UK \\ e-mail:l.krzczanowicz@aston.ac.uk
}

\begin{abstract}
A novel design of a broadband discrete Raman/EDFA hybrid amplifier is proposed. A broadband signal of 84 channels across $75 \mathrm{~nm}$ is experimentally amplified and the gain contribution of each amplifier stage is shown. The amplifier consists of a discrete Raman amplifier using a DCF fibre pumped at three 14xx nm wavelengths, which is followed by a $1480 \mathrm{~nm}$ pumped erbium doped fibre. An average gain of $15.4 \mathrm{~dB}$ and gain flatness of $\pm 1.7 \mathrm{~dB}$ with no gain equalising elements is achieved.
\end{abstract}

Keywords: broadband optical amplification, discrete Raman, Raman/EDFA hybrid amplifier, WDM systems.

\section{INTRODUCTION}

The incredible growth of high speed internet and data traffic creates a demand for increasing the transmission capacity of current wavelength division multiplexed (WDM) based optical communication systems. One way of doing this is enhancing signal bandwidth. Most of the installed fibre links are currently dominated by C-band erbium doped fibre amplifiers (EDFA), whose gain bandwidth is limited (up to $\sim 40 \mathrm{~nm}$ ). In order to surpass this limitation broadband optical amplifiers can be used.

An attractive possibility is to make use of optical fibre based discrete Raman amplifiers, which in principle can have fully configurable gain spectra determined by the Raman gain spectra of multiple pump lasers and scale to more than $100 \mathrm{~nm}$ total bandwidth [1]. Most discrete Raman amplifiers use dispersion compensating fibre (DCF) as the gain medium, which offer simultaneous compensation of loss and dispersion. However, achieving a flat gain spectrum might be very costly as it requires pumping at many wavelengths [2].

A variety of hybrid amplification configurations have been proposed over the years [3, 4], where the EDFA and Raman amplifiers are combined into a serial cascade and each of the stages covers a different part of the $\mathrm{C}+\mathrm{L}$ bands. Avoiding the necessity of band splitters and combiners needed by systems with $\mathrm{C}+\mathrm{L}$ band EDFAs it is possible to simplify the system configuration which decreases the number of loss elements and potentially lowers the overall system cost. The hybrid approach can also reduce the number of pumps used, since the EDFA stage can be pumped with only one pump, which is usually $980 \mathrm{~nm}$ or $1480 \mathrm{~nm}$. It is also possible to improve the total power-to-gain conversion efficiency by using residual Raman pump recycling schemes [5].

In this paper we propose a backward pumped hybrid discrete Raman/EDFA design which consists of an $8 \mathrm{~km}$ DCF, $3 \mathrm{~m}$ erbium doped fibre and five $14 \mathrm{xx} \mathrm{nm}$ pumping units with $200-250 \mathrm{~mW}$ maximum power each. We experimentally amplify a broadband signal of 84 channels with $100 \mathrm{GHz}$ spacing across $75 \mathrm{~nm}$ and obtain an average gain of $15.4 \mathrm{~dB}$ with gain flatness of $\pm 1.7 \mathrm{~dB}$ without using any gain equalising elements. A maximum noise figure of $10.25 \mathrm{~dB}$ was measured, making this amplifier potentially usable in broadband metropolitan WDM networks.

\section{AMPLIFIER DESIGN}

The amplifier design is shown in Fig. 1. It consists of two independent stages separated by an isolator. The first stage is a discrete Raman amplifier consisting of a backward pumped, $8 \mathrm{~km}$ long DCF. The pumping lasers, operated at 1425, 1431 and $1491 \mathrm{~nm}$ are coupled to the fibre with the use of a broadband 14xx pump / C+L band signal WDM combiner and designed to provide gain in the L-band telecom window from $1565 \mathrm{~nm}$ to $1605 \mathrm{~nm}$. The second stage is constructed with a $3 \mathrm{~m}$ long erbium doped fibre which is backward pumped with a $1480 \mathrm{~nm}$ laser diode, giving gain in the C-band $(1530-1565 \mathrm{~nm})$ and is optimised to match the gain value of the first stage.

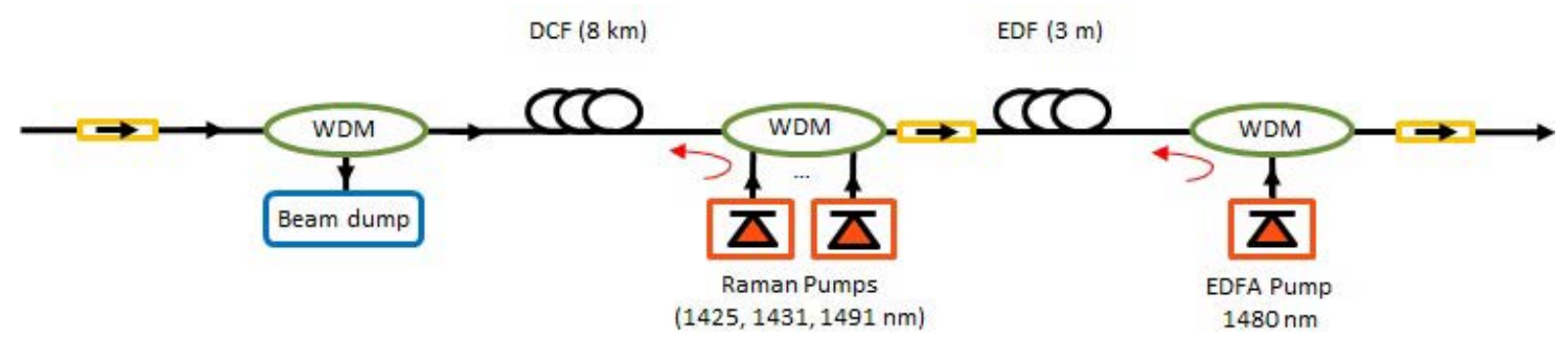

Figure 1. Proposed discrete Raman/EDFA design. 
Since the Raman amplifier should be designed to be a polarisation insensitive device, 1425 and $1431 \mathrm{~nm}$ pumps (single units per wavelength) are combined with an Isolation Hybrid Polarization Beam Combiner and Depolariser (IPBCD). The $1491 \mathrm{~nm}$ pumping consists of two units combined with a Polarisation Beam Combiner (PBC). The $1480 \mathrm{~nm}$ pump of the EDF stage is just a single unit with no special polarisation requirements, which gives us a total of 5 pumps in the amplifier design.

\section{EXPERIMENTAL RESULTS}

The hybrid amplifier performance is analysed in terms of gain, gain ripple and noise figure. The test setup used is shown in Fig. 2. In order to generate a broadband testing signal, C and L band EDFAs were used. Their Amplified Spontaneous Emission (ASE) was shaped with Wavelength Selective Switches (WSS) in order to emulate a WDM transmission signal. Using this approach it was possible to generate 84 channels with $100 \mathrm{GHz}$ spacing across the $\mathrm{C}$ and $\mathrm{L}$ bands from $1529.5 \mathrm{~nm}$ to $1604.8 \mathrm{~nm}$ with a noticeable gap in between the $1560-1570 \mathrm{~nm}$ region. The channels were equalised to be close to $-20 \mathrm{dBm}$ each. There was a fixed attenuator at the OSA input. The acquired spectra were then processed with Matlab software in order to calculate the gain of each channel. The pump powers used for each stage of the amplifier are shown in Table 1.

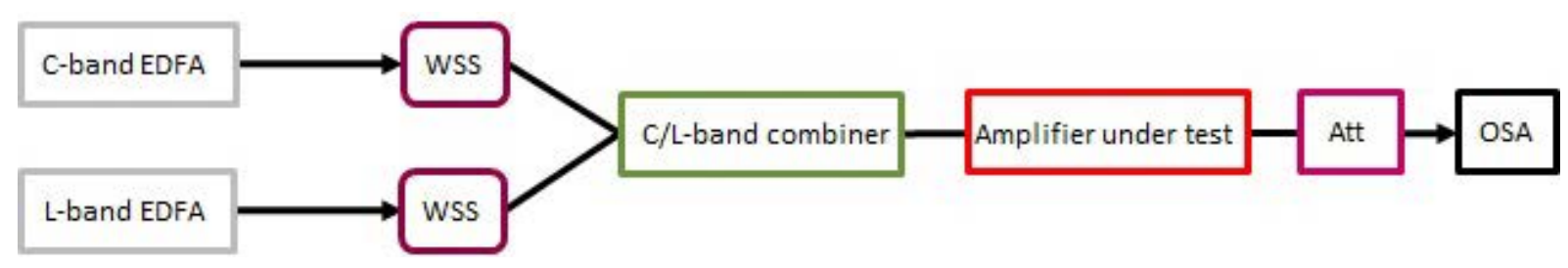

Figure 2. Test setup.

Table 1. Pump values.

\begin{tabular}{|c|c|}
\hline Pump wavelength (nm) & Power (mW) \\
\hline 1425 & 210 \\
\hline 1431 & 180 \\
\hline 1491 & 290 \\
\hline 1480 & 200 \\
\hline
\end{tabular}

Figure 3 shows spectra of all of the 84 input channels and the signal after the first stage of the amplification. It can be seen that the discrete Raman amplifier provides the majority of its gain in the L-band. This might be a little bit counter intuitive since the $1425 \mathrm{~nm}$ and $1431 \mathrm{~nm}$ pumps are used, which would normally result in a high C-band gain spectrum, but in this case a significant pump power transfer occurs intensifying the $1491 \mathrm{~nm}$ Raman shift effect.
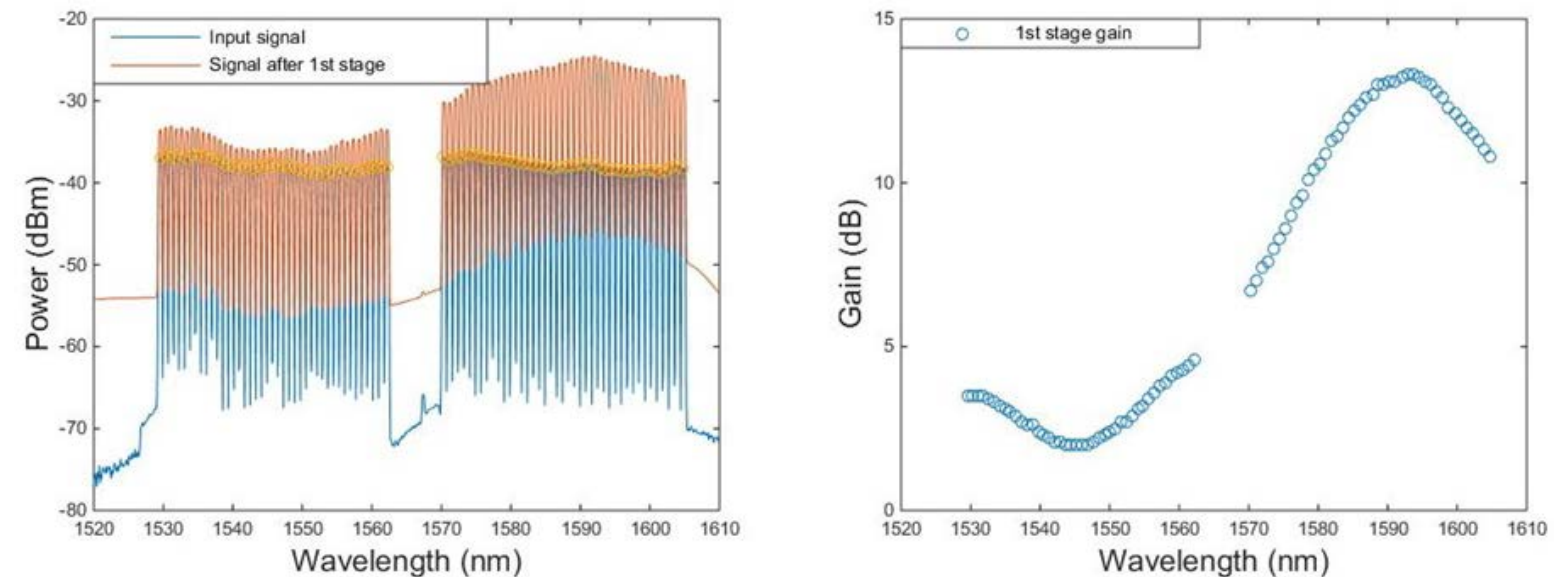

Figure 3. Signal propagation and gain shape after first stage.

Amplification of the second stage can be seen in Fig. 4. The erbium doped fibre (EDF) length was designed in order to provide an average of $13 \mathrm{~dB}$ C-band gain, to match the L-band gain provided by the first stage. Using a $1480 \mathrm{~nm}$ backward pump it is possible to adjust the design of the amplifier in the future so that the residual pump is used to pump the first stage as well. Spectra of the input and fully amplified output signals together with the amplifiers total gain are shown in Fig. 5. The average gain is $15.4 \mathrm{~dB}$ with a gain flatness of $\pm 1.7 \mathrm{~dB}$. 

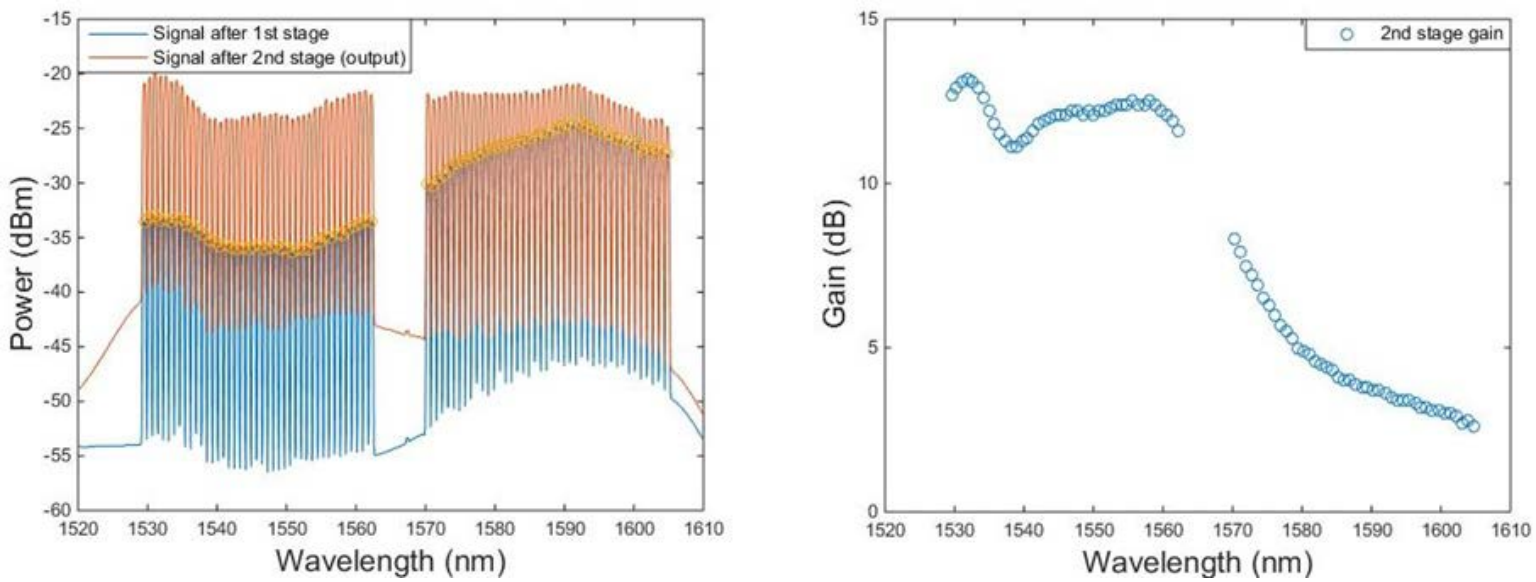

Figure 4. Signal propagation and gain shape after second stage.
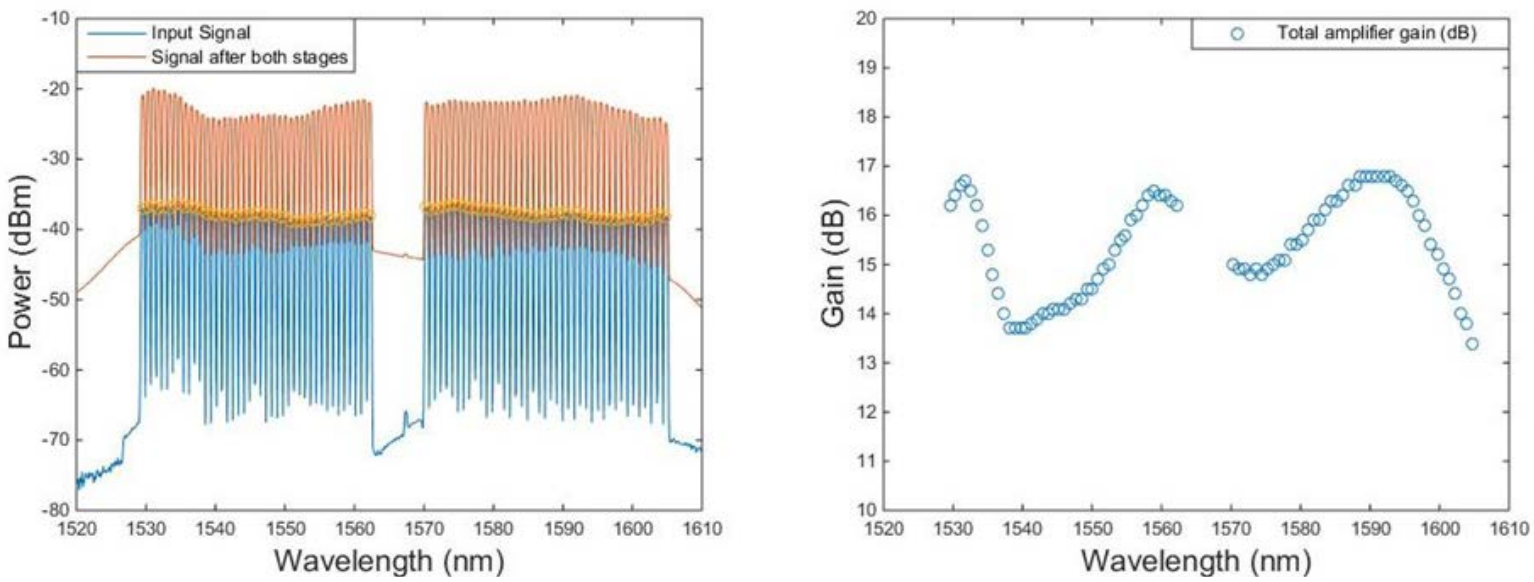

Figure 5. Signal propagation and gain shape after both stages.

In order to measure the noise figure of the amplifier a continuous wave $(\mathrm{CW})$ laser was used. Setting the power to $-20 \mathrm{dBm}$, we measured the input power from $1530 \mathrm{~nm}$ to $1600 \mathrm{~nm}$ with a $5 \mathrm{~nm}$ step and recorded the spectra of the amplified signal. This data was then used to extract the noise figure, which values are shown in Fig. 6.

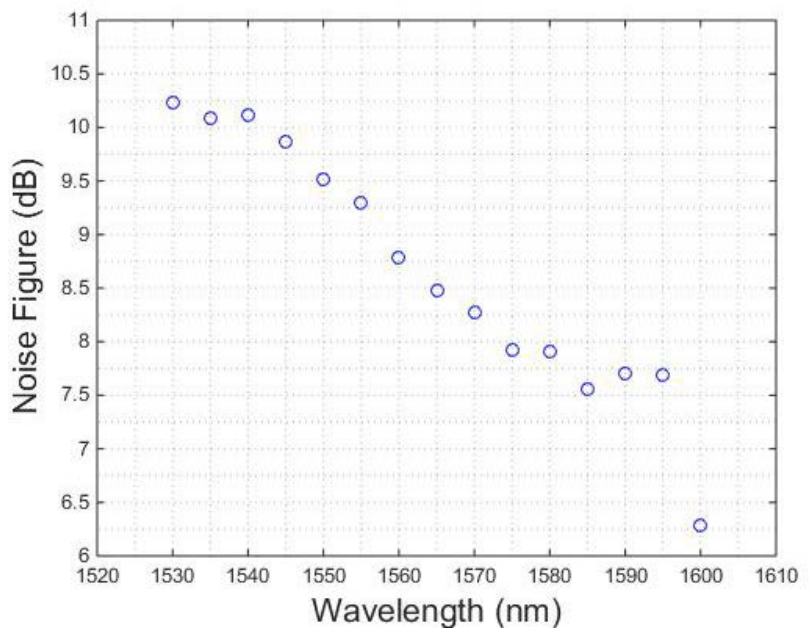

Figure 6. Calculated noise figure.

\section{DISCUSSION}

It is interesting to consider the suitability of this amplifier for use in future broadband metropolitan WDM networks and for data centre interconnect (DCI). While the hybrid discrete Raman/EDFA described here increases bandwidth from $\sim 40 \mathrm{~nm}$ to $\sim 75 \mathrm{~nm}$, nearly doubling the available capacity, its output power, gain and gain ripple fall well short of present EDFA specifications, which can provide output power of up to $23 \mathrm{dBm}$, variable gain up to $40 \mathrm{~dB}$ and gain ripple better than $1 \mathrm{~dB}[6]$. 
It is clear that gain ripple could be reduced using a suitable gain flattening filter, while output power and gain could be increased further with the use of higher power 14xx Raman pump lasers with individual output powers of up to $600 \mathrm{~mW}[7,8]$ and highly compact $980 \mathrm{~nm}$ pumps with powers of up to $1 \mathrm{~W}$ [9]. Higher pump power would also reduce the length of DCF required, which together with direct availability of high power $\sim 1490 \mathrm{~nm}$ pumps (rather than the pump combination used here transferring power from 1425/31 nm to $1491 \mathrm{~nm}$, the pump lasers available in our laboratory) could reduce the pump count from 5 to 3 . Alternatively, a further pair of Raman pump lasers, at say $1510 \mathrm{~nm}$, could potentially increase the gain bandwidth to over $100 \mathrm{~nm}$, thus enabling continuous amplification of the C-band and an extended L-band.

Nevertheless, a number of significant technical challenges remain to be solved before the discrete hybrid Raman/EDFA becomes a commercially attractive proposition for system designers. In particular, the use of long lengths of DCF and the relative inefficiency of the Raman amplification process leads to increased space requirements and decreased power efficiency. While there is no immediate prospect of a highly nonlinear fibre offering two orders of magnitude increase in Raman efficiency, which would address both these issues, the advent of cost-effective $100 \mathrm{Gbit} / \mathrm{s}$ interfaces based on direct-detection transceivers for data centre networking [10] could take advantage of the DCF in a hybrid Raman/EDFA for simultaneous dispersion compensation, removing the need to use coherent interfaces, and might therefore be attractive in the overall system design.

\section{CONCLUSIONS}

We have proposed a novel design of discrete broadband Raman/EDFA amplifier capable of amplifying a WDM broadband transmission. The amplifier consists of a discrete Raman amplifier using a DCF fibre pumped at three $14 \mathrm{xx} \mathrm{nm}$ wavelengths, followed by a $1480 \mathrm{~nm}$ pumped EDFA. 84 simultaneous channels across $75 \mathrm{~nm}$ with average power of $-20 \mathrm{dBm}$ per channel were amplified. The average gain of $15.4 \mathrm{~dB}$ with gain flatness of $\pm 1.7 \mathrm{~dB}$ over $75 \mathrm{~nm}$ is achieved. No gain equalising elements were used. The noise figure with its maximum value of $10.25 \mathrm{~dB}$ makes this amplifier an interesting candidate for broadband metro WDM networks and DCIs, where the noise figure requirements are more relaxed due to the relatively short transmission lengths.

\section{ACKNOWLEDGEMENTS}

This work has been supported by the EPSRC project EP/M009092/1. We thank II-VI for support and helpful discussions.

\section{REFERENCES}

[1] S. Namiki and Y. Emori, "Ultrabroad-band Raman amplifiers pumped and gain-equalized by wavelengthdivision-multiplexed high-power laser diodes," IEEE Journal of Selected Topics in Quantum Electronics, vol. 7, no. 1, pp. 3-16, Jan./Feb. 2001.

[2] Y. Emori and S. Namiki, "100 nm bandwidth flat gain Raman amplifiers pumped and gain-equalized by 12-wavelength-channel WDM high power laser diodes," in Proc. OFC/IOOC, San Diego, CA, USA, 1999, pp. PD19/1-PD19/3.

[3] H. Masuda and S. Kawai, "Wide-band and gain-flattened hybrid fiber amplifier consisting of an EDFA and a multiwavelength pumped Raman amplifier," IEEE Photonics Technology Letters, vol. 11, no. 6, pp. 647649, Jun. 1999.

[4] B. Zhu et al., "Large-area low-loss fibers and advanced amplifiers for high-capacity long-haul optical networks [Invited]," Journal of Optical Communications and Networking, vol. 8, no. 7, pp. A55-A63, 2016.

[5] M. M. J. Martini, C. E. S. Castellani, M. J. Pontes, M. R. N. Ribeiro, and H. J. Kalinowski, "Performance comparison for Raman+EDFA and EDFA+Raman hybrid amplifiers using recycled multiple pump lasers for WDM systems," in Proc. SBMO/IEEE MTT-S International Microwave and Optoelectronics Conference (IMOC), Porto de Galinhas, 2015, pp. 1-5.

[6] G. Cowle, "The state of the art of modern non-SDM amplification technology in agile optical networks: EDFA and Raman amplifiers and circuit packs," in Proc. OFC, Washington, DC, 2017, paper M3G.1.

[7] https://www.anritsu.com/en-US/anritsu-devices/products/fp-ld-series

[8] https://www.lumentum.com/en/optical-communications/products/pump-lasers/14xx-pump-lasers

[9] http://www.ii-vi-photonics.com/products/amplifier-components.html

[10] D.V. Plant, M. Morsy-Osman, and M. Chagnon, "Optical communication systems for datacenter networks," in Proc. OFC, Washington, DC, 2017, paper W3B.1. 